fractions confirmed that $\beta$-adrenergic stimulation by isoproterenol decreased the B56 $\alpha$ content of the myofilament fraction in the absence of significant changes in the myosin phosphatase target subunit isoforms 1 and 2 (MYPT1 and MYPT2). Furthermore, immunolabelling and confocal microscopy revealed the spatial redistribution of these proteins, with a loss of B56 $\alpha$ from Z-disc and $\mathrm{M}$-band regions but increased association of MYPT1/2 with A-band regions of the sarcomere, following $\beta$-adrenergic stimulation.

Conclusion We present the first comprehensive proteomic dataset of skinned cardiomyocytes and demonstrate the potential of proteomics to unravel dynamic changes in protein composition that may contribute to the neurohormonal regulation of myofilament contraction.

\section{YIA4 C JUN N-TERMINAL KINASE PROMOTES ENDOTHELIAL ACTIVATION AT ATHEROSCLEROSIS-SUSCEPTIBLE SITES BY ENHANCING EXPRESSION OF NF-KB TRANSCRIPTION FACTORS}

doi:10.1136/hrt.2010.205781.4

\begin{abstract}
${ }^{1,2}$ Simon Cuhlmann, ${ }^{1}$ Kim van der Heiden, ${ }^{1}$ Mustafa Zakkar, ${ }^{1}$ Hera Chaudhury, ${ }^{1}$ Le Anh Luong, ' Justin Mason, 'Dorian 0 Haskard, ${ }^{3}$ Harald Carlsen, ${ }^{2}$ Robert Krams, ${ }^{1}$ Paul C Evans. ${ }^{1}$ BHF Cardiovascular Sciences Unit, National Heart and Lung Institute, Imperial College London, UK; ${ }^{2}$ Department of Bioengineering, Imperial College London, UK; ${ }^{3}$ Department of Nutrition, University of Oslo, Norway
\end{abstract}

Atherosclerosis develops predominantly at branches and bends in arteries that are exposed to disturbed flow which exerts low, oscillatory shear stress on endothelial cells (ECs). We demonstrated that c-Jun N-terminal kinase (JNK) is activated in ECs at atherosusceptible but not atheroprotected sites. Transcriptome profiling of cultured ECs treated with a pharmacological inhibitor revealed that JNK functions as a positive regulator of NF- $\mathrm{B}$ transcription factors, which promote inflammation by inducing inflammatory molecules (eg, VCAM-1). This observation was confirmed by silencing of JNK1 and ATF2 (a downstream transcription factor), which led to reduced NF- $\kappa B$ expression in cultured ECs. We validated our findings by demonstrating that EC expression of NF- $\mathrm{KB}$ and VCAM-1 and the accumulation of CD68-positive macrophages was elevated at atherosusceptible sites compared with atheroprotected sites in aortas of wild-type mice. Genetic deletion of JNK1 suppressed NF- $\kappa B$ and VCAM-1 expression, and reduced macrophage accumulation at the atherosusceptible site, indicating that JNK1 positively regulates NF$\kappa \mathrm{B}$ expression and inflammation. To establish a causal relationship between shear stress and JNK activity, we altered blood flow in the murine carotid artery by placing a constrictive cuff. We observed that low, oscillatory shear stress can enhance JNK activity, NF- $\mathrm{KB}$ and VCAM-1 expression in ECs and promote macrophage accumulation in arteries. We conclude that JNK1-ATF2 signalling promotes EC activation and inflammation at atheroprone sites exposed to low, oscillatory shear stress by enhancing NF- $\kappa B$ expression. Our findings illuminate a novel level of cross-talk between the NF-KB and JNK signalling pathways that may influence the spatial distribution of atherosclerotic lesions.

Funding British Heart Foundation and NHLI Trustees Foundation.

\section{YIA5 PKC $\delta$ DEFICIENCY ACCELERATES NEOINTIMAL LESIONS IN A MOUSE MODEL OF VASCULAR INJURY INVOLVING DELAYED RE-ENDOTHELIALISATION AND VASOHIBIN-1 ACCUMULATION}

doi:10.1136/hrt.2010.205781.5

Xue Bai, Andriana Margariti, Lingfang Zeng, Yanhua Hu, Qingbo Xu. Cardiovascular Division, King's College London BHF Centre, London, UK

Objective Protein kinase C (PKC) $\delta$ functions as a signal transducer mediating several essential functions of cell proliferation and apoptosis. However, the effect of $\mathrm{PKC} \delta$ on neointimal formation is currently unknown. In this study, we used a vascular injury model in PKC $\delta$ knockout mice to investigate the role of PKC $\delta$ in the lesion development and underlying mechanism.

Methods and results 3 weeks after wire injury of femoral arteries, neointimal lesions were significantly increased in $\mathrm{PKC} \delta-/-$ mice compared with wild-type animals. Immunohistochemical staining revealed that total numbers of smooth muscle cells and macrophages in the lesions were markedly elevated without the alterations of the ratio between these two types of cells. To further study the mechanisms of $\mathrm{PKC} \delta$-mediated increase in the lesion, an in vivo endothelial migration model was established to evaluate endothelial wound healing after wire injury. Data indicate that re-endothelialisation of the injured vessel was markedly delayed in PKC $\delta-/-$ mice that coincided with more severe intimal hyperplasia. When endothelial cells were cultivated from cardiac tissues of $\mathrm{PKC} \delta-/-$ and PKC $\delta+/+$ mice, the ability of cell migration was significantly reduced in $\mathrm{PKC} \delta-/-$ mice, but no difference in proliferation and apoptosis was found. This altered endothelial migration was also verified by PKC $\delta$ inhibitor and siRNA techniques in wild-type cells. Interestingly, vasohibin-1, an anti-migration protein, was elevated in endothelial cells derived from PKC $\delta$-deficient mice, which was identified largely owing to delayed protein degradation mediated by $\mathrm{PKC} \delta$. Downregulation of vasohibin-1 restored the migration rate of PKC $\delta-/$ - endothelial cells to a level similar to that in wild-type cells. Conclusion Our data provide the first evidence that PKC $\delta$-enhanced neointima formation occurs mainly due to delayed re-endothelialisation, which is mediated by increased cellular vasohibin- 1 that is regulated by $\mathrm{PKC} \delta$.

\section{YIA6 EFFECTS OF GLP-1 ELUTING STEM CELL THERAPY ON COLLAGEN REMODELLING, INFARCT SIZE AND APOPTOSIS IN A PORCINE MODEL OF MYOCARDIAL INFARCTION}

doi:10.1136/hrt.2010.205781.6

${ }^{1} \mathrm{E}$ J Wright, ${ }^{1} \mathrm{~K}$ A Farrell, ${ }^{2} \mathrm{C}$ Wallrapp, ${ }^{2} \mathrm{P}$ Geigle, ${ }^{3} \mathrm{~A}$ L Lewis, ${ }^{3} \mathrm{P}$ W Stratford, ${ }^{1} \mathrm{~N}$ Malik, ${ }^{1} \mathrm{C}$ M Holt. ${ }^{1}$ Cardiovascular Research Group, University of Manchester, UK; ${ }^{2} \mathrm{CellMed}$ AG, Alzenau, Germany; ${ }^{3}$ Biocompatibles UK Ltd, Surrey, UK

Glucagon-like peptide-1 (GLP-1) is a gut incretin hormone with cardioprotective effects. Human stem cells secreting a GLP-1 fusion protein and encapsulated in an alginate matrix (GLP-1 CellBeads) have been developed as a novel therapeutic agent. This study investigated the effects of GLP-1 CellBeads on post-myocardial infarction (MI) healing in a porcine model. GLP-1 CellBeads were delivered to the left anterior descending coronary artery to create micro-infarcts, with cell-free beads as controls. Hearts were explanted at 1 and 4 weeks post-MI. Gross infarct size was measured as a percentage of left ventricular area. Tissue was analysed for inflammation (number of MAC 387 positive cells $/ \mathrm{mm}^{2}$ ), apoptosis (\% TUNEL positive cells) and collagen (\% picrosirius red staining).

Compared with controls $(n=4)$, the GLP-1-treated group $(n=6)$ exhibited less infarct at one $(6.21 \% \pm 0.64$ vs $9.78 \% \pm 1.80 \% 2 \mathrm{LV}$, $\mathrm{p}=\mathrm{NS})$ and 4 weeks post-MI $(4.7 \% \pm 2.1$ vs $21.8 \% \pm 4.8 \% \mathrm{LV}$, $\mathrm{p}=0.02)$. Within the infarct there was increased inflammation in GLP-1-treated groups at both time points (1 week: $97.22 \% \pm 19.62$ vs $36.67 \% \pm 7.78, \quad p=0.01 ; 4$ weeks: $24.2 \% \pm 4.57$ vs $12.3 \% \pm 3.16$, $\mathrm{p}=0.03)$. At 1 week, apoptosis rates in the infarct area were similar in both groups $(1.46 \% \pm 0.57$ vs $1.47 \% \pm 0.17, p=N S)$, with less apoptosis in the GLP-1-treated group at 4 weeks $(0.51 \% \pm 0.18$ vs $1.84 \% \pm 0.62, p=N S)$. Collagen content at 1 week was lower $(5.14 \% \pm 1.19$ vs $9.95 \% \pm 1.42, \mathrm{p}=0.05)$ and at 4 weeks higher in the treated group $(20.89 \% \pm 8.25$ vs $6.87 \% \pm 2.92, p=N S)$. GLP-1 CellBeads have an effect on post-MI infarct size, inflammation and 